\title{
Aromaticity of diazaborines and their protonated forms
}

\author{
Milovan Stojanović, ${ }^{\dagger}$ and Marija Baranac-Stojanović, ${ }^{*+}$ \\ ${ }^{\dagger}$ Center for Chemistry ICTM, University of Belgrade, Njegoševa 12, P.O.Box 473, 11000 Belgrade, Serbia \\ ${ }^{\ddagger}$ Faculty of Chemistry, University of Belgrade, Studentski trg 12-16, P.O.Box 158, 11000 Belgrade, Serbia
}

\section{Supporting Information}

\section{Table of Contents}

Table S1. Calculated bond lengths of 6-15 and 6-15- $\mathrm{H}^{+}$

Absolute energies and $\mathrm{x}, \mathrm{y}, \mathrm{z}$ coordinates of diazaborines

and their protonated forms.......

S4 
Table S1. Calculated bond lengths of 6-15 and 6-15- $\mathrm{H}^{+}$.

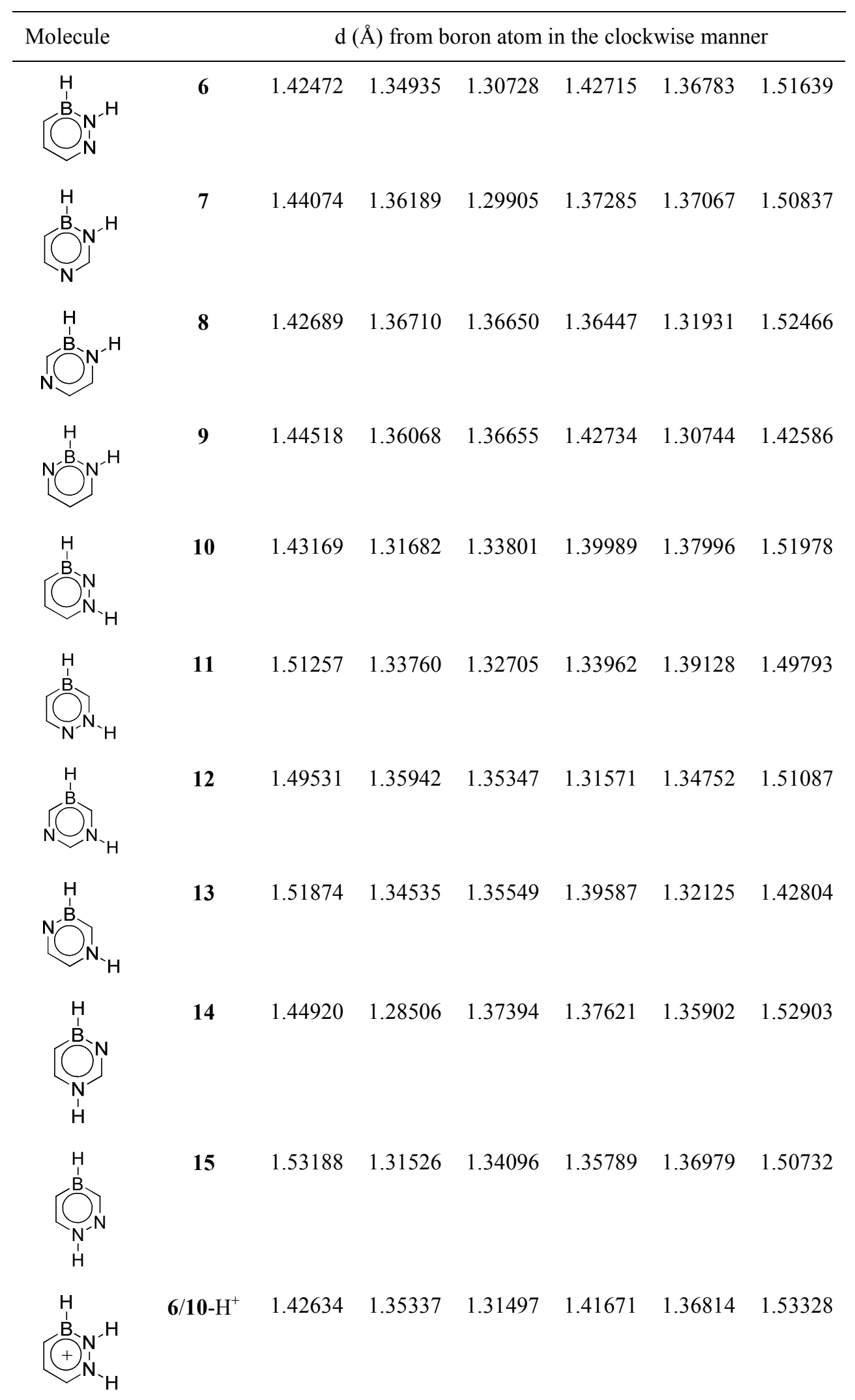




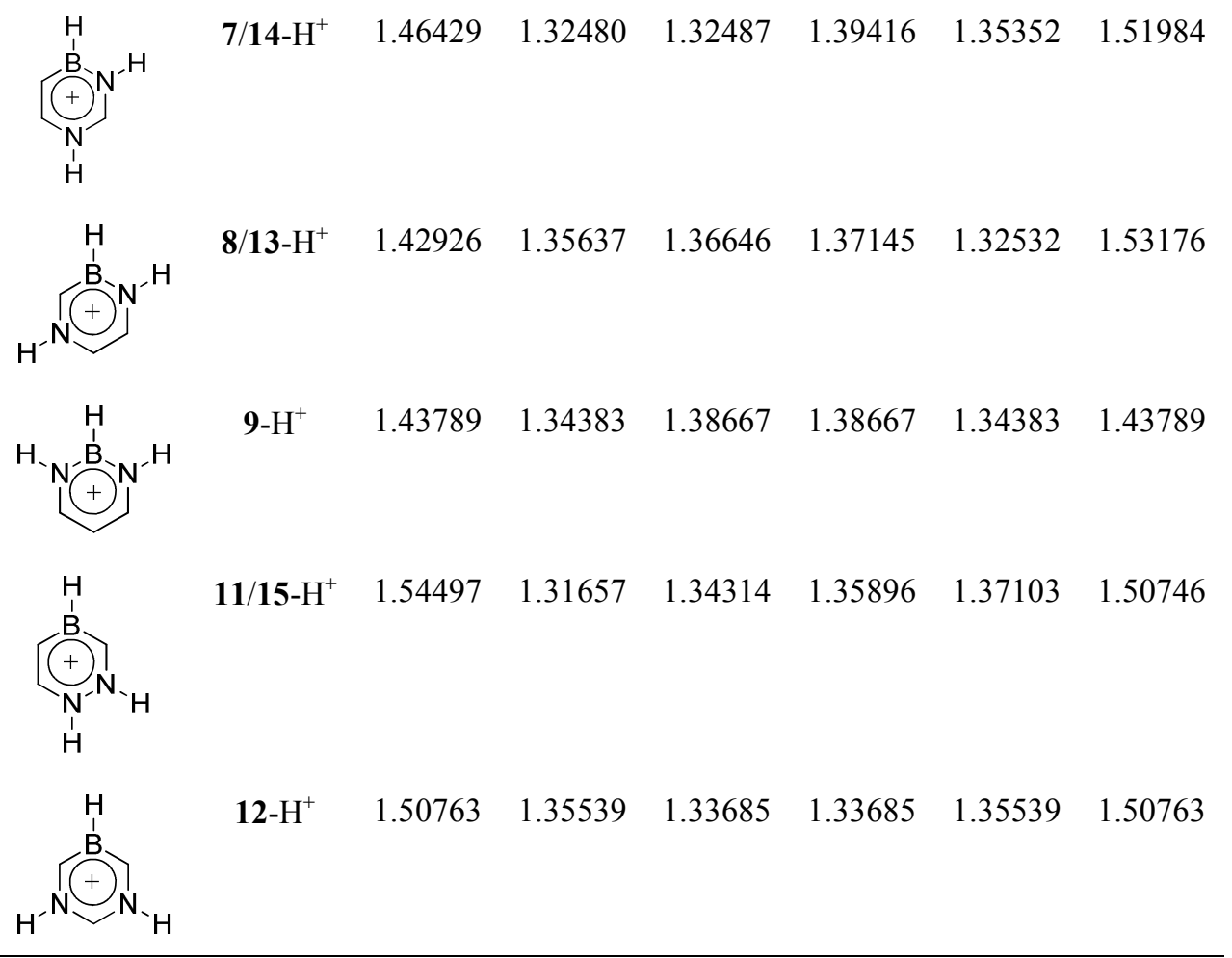




\section{Absolute energies (a.u.) and $x, y, z$ coordinates ( $\AA$ ) of diazaborines and their protonated forms}

6, $\mathrm{E}=-251.7653724$ a.u.

\begin{tabular}{|c|c|c|c|c|c|}
\hline \multirow{2}{*}{$\begin{array}{l}\text { Center } \\
\text { Number }\end{array}$} & \multirow{2}{*}{$\begin{array}{l}\text { Atomic } \\
\text { Number }\end{array}$} & \multirow{2}{*}{$\begin{array}{l}\text { Atomic } \\
\text { Type }\end{array}$} & \multicolumn{3}{|c|}{ Coordinates (Angstroms) } \\
\hline & & & $\mathrm{X}$ & $\mathrm{Y}$ & Z \\
\hline 1 & 6 & 0 & 1.241241 & 0.623236 & 0.000000 \\
\hline 2 & 6 & 0 & 0.000000 & 1.327565 & 0.000000 \\
\hline 3 & 7 & 0 & -1.194977 & -0.585400 & 0.000000 \\
\hline 4 & 1 & 0 & 2.152142 & 1.216219 & 0.000000 \\
\hline 5 & 1 & 0 & -0.016542 & 2.412184 & 0.000000 \\
\hline 6 & 5 & 0 & -0.071079 & -1.461010 & 0.000000 \\
\hline 7 & 1 & 0 & -0.276306 & -2.631856 & 0.000000 \\
\hline 8 & 1 & 0 & -2.146532 & -0.927210 & 0.000000 \\
\hline 9 & 6 & 0 & 1.265286 & -0.744381 & 0.000000 \\
\hline 10 & 1 & 0 & 2.224776 & -1.252052 & 0.000000 \\
\hline 11 & 7 & 0 & -1.179495 & 0.763863 & 0.000000 \\
\hline
\end{tabular}

7, $\mathrm{E}=-251.7938367$ a.u.

\begin{tabular}{|c|c|c|c|c|c|}
\hline \multirow{2}{*}{$\begin{array}{l}\text { Center } \\
\text { Number }\end{array}$} & \multirow{2}{*}{$\begin{array}{l}\text { Atomic } \\
\text { Number }\end{array}$} & \multirow{2}{*}{$\begin{array}{c}\text { Atomic } \\
\text { Type }\end{array}$} & \multicolumn{3}{|c|}{ Coordinates (Angstroms) } \\
\hline & & & $\mathrm{X}$ & $\mathrm{Y}$ & Z \\
\hline 1 & 6 & 0 & -1.221365 & -0.617654 & 0.000000 \\
\hline 2 & 7 & 0 & 1.180555 & 0.636997 & 0.000000 \\
\hline 3 & 1 & 0 & -2.233910 & -1.015084 & 0.000000 \\
\hline 4 & 5 & 0 & 1.237419 & -0.802616 & 0.000000 \\
\hline 5 & 1 & 0 & 2.302748 & -1.329935 & 0.000000 \\
\hline 6 & 1 & 0 & 2.016729 & 1.206576 & 0.000000 \\
\hline 7 & 6 & 0 & -0.127797 & -1.443998 & 0.000000 \\
\hline 8 & 1 & 0 & -0.299701 & -2.515390 & 0.000000 \\
\hline 9 & 1 & 0 & 0.057448 & 2.401829 & 0.000000 \\
\hline 10 & 6 & 0 & 0.000000 & 1.316001 & 0.000000 \\
\hline 11 & 7 & 0 & -1.171331 & 0.754288 & 0.000000 \\
\hline
\end{tabular}


8, $\mathrm{E}=-251.7779872$ a.u.

\begin{tabular}{|c|c|c|c|c|c|}
\hline \multirow{2}{*}{$\begin{array}{l}\text { Center } \\
\text { Number }\end{array}$} & \multirow{2}{*}{$\begin{array}{l}\text { Atomic } \\
\text { Number }\end{array}$} & \multirow{2}{*}{$\begin{array}{l}\text { Atomic } \\
\text { Type }\end{array}$} & \multicolumn{3}{|c|}{ Coordinates (Angstroms) } \\
\hline & & & $\mathrm{X}$ & Y & Z \\
\hline 1 & 7 & 0 & 1.189113 & 0.658839 & 0.000000 \\
\hline 2 & 1 & 0 & -2.117646 & 1.172289 & 0.000000 \\
\hline 3 & 5 & 0 & 1.250859 & -0.766715 & 0.000000 \\
\hline 4 & 1 & 0 & 2.309056 & -1.307138 & 0.000000 \\
\hline 5 & 1 & 0 & 2.026725 & 1.225420 & 0.000000 \\
\hline 6 & 6 & 0 & -0.126729 & -1.420034 & 0.000000 \\
\hline 7 & 1 & 0 & -0.269933 & -2.500777 & 0.000000 \\
\hline 8 & 1 & 0 & 0.028069 & 2.416178 & 0.000000 \\
\hline 9 & 6 & 0 & 0.000000 & 1.333354 & 0.000000 \\
\hline 10 & 6 & 0 & -1.175413 & 0.636406 & 0.000000 \\
\hline 11 & 7 & 0 & -1.248787 & -0.726089 & 0.000000 \\
\hline
\end{tabular}

9, $\mathrm{E}=-251.8041313$ a.u.

\begin{tabular}{|c|c|c|c|c|c|}
\hline \multirow{2}{*}{$\begin{array}{l}\text { Center } \\
\text { Number }\end{array}$} & \multirow{2}{*}{$\begin{array}{l}\text { Atomic } \\
\text { Number }\end{array}$} & \multirow{2}{*}{$\begin{array}{c}\text { Atomic } \\
\text { Type }\end{array}$} & \multicolumn{3}{|c|}{ Coordinates (Angstroms) } \\
\hline & & & $\mathrm{X}$ & Y & Z \\
\hline 1 & 6 & 0 & -0.107181 & -1.370417 & 0.000000 \\
\hline 2 & 6 & 0 & 1.157873 & -0.709397 & 0.000000 \\
\hline 3 & 6 & 0 & 1.174399 & 0.657056 & 0.000000 \\
\hline 4 & 7 & 0 & -1.265882 & -0.764773 & 0.000000 \\
\hline 5 & 1 & 0 & 2.094465 & 1.230767 & 0.000000 \\
\hline 6 & 1 & 0 & -0.098927 & -2.464640 & 0.000000 \\
\hline 7 & 1 & 0 & 2.082702 & -1.271269 & 0.000000 \\
\hline 8 & 1 & 0 & 0.060809 & 2.353390 & 0.000000 \\
\hline 9 & 1 & 0 & -2.260902 & 1.326609 & 0.000000 \\
\hline 10 & 5 & 0 & -1.273504 & 0.661071 & 0.000000 \\
\hline 11 & 7 & 0 & 0.000000 & 1.344249 & 0.000000 \\
\hline
\end{tabular}


10, $E=-251.7348337$ a.u.

\begin{tabular}{|c|c|c|c|c|c|}
\hline \multirow{2}{*}{$\begin{array}{l}\text { Center } \\
\text { Number }\end{array}$} & \multirow{2}{*}{$\begin{array}{l}\text { Atomic } \\
\text { Number }\end{array}$} & \multirow{2}{*}{$\begin{array}{c}\text { Atomic } \\
\text { Type }\end{array}$} & \multicolumn{3}{|c|}{ Coordinates (Angstroms) } \\
\hline & & & $\mathrm{X}$ & $\mathrm{Y}$ & Z \\
\hline 1 & 6 & 0 & -1.150038 & -0.789086 & 0.000000 \\
\hline 2 & 6 & 0 & -1.160802 & 0.610766 & 0.000000 \\
\hline 3 & 7 & 0 & 1.222828 & 0.787632 & 0.000000 \\
\hline 4 & 1 & 0 & -2.107026 & -1.301788 & 0.000000 \\
\hline 5 & 1 & 0 & -2.059735 & 1.212845 & 0.000000 \\
\hline 6 & 5 & 0 & 1.339615 & -0.639289 & 0.000000 \\
\hline 7 & 1 & 0 & 2.436876 & -1.104198 & 0.000000 \\
\hline 8 & 6 & 0 & 0.057998 & -1.456113 & 0.000000 \\
\hline 9 & 1 & 0 & 0.034929 & -2.542785 & 0.000000 \\
\hline 10 & 7 & 0 & 0.000000 & 1.276202 & 0.000000 \\
\hline 11 & 1 & 0 & -0.045868 & 2.292135 & 0.000000 \\
\hline
\end{tabular}

11, $\mathrm{E}=-251.7255578$ a.u.

\begin{tabular}{|c|c|c|c|c|c|}
\hline \multirow{2}{*}{$\begin{array}{l}\text { Center } \\
\text { Number }\end{array}$} & \multirow{2}{*}{$\begin{array}{l}\text { Atomic } \\
\text { Number }\end{array}$} & \multirow{2}{*}{$\begin{array}{c}\text { Atomic } \\
\text { Type }\end{array}$} & \multicolumn{3}{|c|}{ Coordinates (Angstroms) } \\
\hline & & & $\mathrm{X}$ & Y & Z \\
\hline 1 & 5 & 0 & 1.310104 & -0.812390 & 0.000000 \\
\hline 2 & 1 & 0 & 2.358532 & -1.375764 & 0.000000 \\
\hline 3 & 1 & 0 & -0.220870 & -2.525069 & 0.000000 \\
\hline 4 & 6 & 0 & 1.207430 & 0.696691 & 0.000000 \\
\hline 5 & 1 & 0 & 2.017393 & 1.420782 & 0.000000 \\
\hline 6 & 1 & 0 & -2.181338 & -1.067485 & 0.000000 \\
\hline 7 & 6 & 0 & -0.044095 & -1.452645 & 0.000000 \\
\hline 8 & 6 & 0 & -1.178944 & -0.647802 & 0.000000 \\
\hline 9 & 7 & 0 & -1.193332 & 0.691736 & 0.000000 \\
\hline 10 & 7 & 0 & 0.000000 & 1.272267 & 0.000000 \\
\hline 11 & 1 & 0 & -0.077256 & 2.283999 & 0.000000 \\
\hline
\end{tabular}

12, $\mathrm{E}=-251.7385302$ a.u.

\begin{tabular}{|c|c|c|c|c|c|}
\hline \multirow{2}{*}{$\begin{array}{l}\text { Center } \\
\text { Number }\end{array}$} & \multirow{2}{*}{$\begin{array}{l}\text { Atomic } \\
\text { Number }\end{array}$} & \multirow{2}{*}{$\begin{array}{c}\text { Atomic } \\
\text { Type }\end{array}$} & \multicolumn{3}{|c|}{ Coordinates (Angstroms) } \\
\hline & & & $\mathrm{X}$ & $\mathrm{Y}$ & Z \\
\hline 1 & 6 & 0 & -1.161326 & 0.585326 & 0.000000 \\
\hline 2 & 6 & 0 & 1.228640 & 0.698668 & 0.000000 \\
\hline 3 & 6 & 0 & -0.066331 & -1.428071 & 0.000000 \\
\hline 4 & 7 & 0 & -1.218417 & -0.729145 & 0.000000 \\
\hline 5 & 1 & 0 & 2.044446 & 1.414312 & 0.000000 \\
\hline 6 & 1 & 0 & -2.072546 & 1.172132 & 0.000000 \\
\hline 7 & 1 & 0 & -0.074568 & 2.291774 & 0.000000 \\
\hline 8 & 1 & 0 & 2.333433 & -1.394335 & 0.000000 \\
\hline 9 & 1 & 0 & -0.234572 & -2.505230 & 0.000000 \\
\hline 10 & 7 & 0 & 0.000000 & 1.280458 & 0.000000 \\
\hline 11 & 5 & 0 & 1.305365 & -0.794677 & 0.000000 \\
\hline
\end{tabular}


13, $E=-251.7496744$ a.u.

\begin{tabular}{|c|c|c|c|c|c|}
\hline \multirow{2}{*}{$\begin{array}{l}\text { Center } \\
\text { Number }\end{array}$} & \multirow{2}{*}{$\begin{array}{l}\text { Atomic } \\
\text { Number }\end{array}$} & \multirow{2}{*}{$\begin{array}{c}\text { Atomic } \\
\text { Type }\end{array}$} & \multicolumn{3}{|c|}{ Coordinates (Angstroms) } \\
\hline & & & $\mathrm{X}$ & $\mathrm{Y}$ & Z \\
\hline 1 & 7 & 0 & 0.003153 & -1.489712 & 0.000000 \\
\hline 2 & 1 & 0 & -0.059434 & 2.307366 & 0.000000 \\
\hline 3 & 1 & 0 & -2.083140 & 1.186842 & 0.000000 \\
\hline 4 & 5 & 0 & 1.257922 & -0.807904 & 0.000000 \\
\hline 5 & 1 & 0 & 2.296477 & -1.396383 & 0.000000 \\
\hline 6 & 6 & 0 & 1.211652 & 0.710128 & 0.000000 \\
\hline 7 & 1 & 0 & 2.040229 & 1.410708 & 0.000000 \\
\hline 8 & 1 & 0 & -2.070934 & -1.313189 & 0.000000 \\
\hline 9 & 6 & 0 & -1.114531 & -0.785088 & 0.000000 \\
\hline 10 & 6 & 0 & -1.169601 & 0.609695 & 0.000000 \\
\hline 11 & 7 & 0 & 0.000000 & 1.294822 & 0.000000 \\
\hline
\end{tabular}

14, $E=-251.7728654$ a.u.

\begin{tabular}{|c|c|c|c|c|c|}
\hline \multirow{2}{*}{$\begin{array}{l}\text { Center } \\
\text { Number }\end{array}$} & \multirow{2}{*}{$\begin{array}{l}\text { Atomic } \\
\text { Number }\end{array}$} & \multirow{2}{*}{$\begin{array}{c}\text { Atomic } \\
\text { Type }\end{array}$} & \multicolumn{3}{|c|}{ Coordinates (Angstroms) } \\
\hline & & & $\mathrm{X}$ & Y & Z \\
\hline 1 & 6 & 0 & 1.207861 & 0.605935 & 0.000000 \\
\hline 2 & 7 & 0 & -1.303702 & -0.696398 & 0.000000 \\
\hline 3 & 1 & 0 & 2.078066 & 1.254349 & 0.000000 \\
\hline 4 & 1 & 0 & -0.015954 & 2.275193 & 0.000000 \\
\hline 5 & 5 & 0 & -0.093621 & -1.493826 & 0.000000 \\
\hline 6 & 1 & 0 & -0.175385 & -2.684586 & 0.000000 \\
\hline 7 & 6 & 0 & 1.243743 & -0.752615 & 0.000000 \\
\hline 8 & 1 & 0 & 2.221042 & -1.223928 & 0.000000 \\
\hline 9 & 1 & 0 & -2.065786 & 1.241154 & 0.000000 \\
\hline 10 & 6 & 0 & -1.192931 & 0.583874 & 0.000000 \\
\hline 11 & 7 & 0 & 0.000000 & 1.265510 & 0.000000 \\
\hline
\end{tabular}

15, $\mathrm{E}=-251.7292137$ a.u.

\begin{tabular}{|c|c|c|c|c|c|}
\hline \multirow{2}{*}{$\begin{array}{l}\text { Center } \\
\text { Number }\end{array}$} & \multirow{2}{*}{$\begin{array}{l}\text { Atomic } \\
\text { Number }\end{array}$} & \multirow{2}{*}{$\begin{array}{c}\text { Atomic } \\
\text { Type }\end{array}$} & \multicolumn{3}{|c|}{ Coordinates (Angstroms) } \\
\hline & & & $\mathrm{X}$ & $\mathrm{Y}$ & Z \\
\hline 1 & 1 & 0 & -0.017770 & 2.263079 & 0.000000 \\
\hline 2 & 5 & 0 & -0.050335 & -1.527033 & 0.000000 \\
\hline 3 & 1 & 0 & -0.145750 & -2.714382 & 0.000000 \\
\hline 4 & 1 & 0 & 2.244366 & -1.212604 & 0.000000 \\
\hline 5 & 6 & 0 & -1.277729 & -0.610425 & 0.000000 \\
\hline 6 & 1 & 0 & -2.307589 & -0.963318 & 0.000000 \\
\hline 7 & 1 & 0 & 2.051563 & 1.257716 & 0.000000 \\
\hline 8 & 6 & 0 & 1.253364 & -0.770469 & 0.000000 \\
\hline 9 & 6 & 0 & 1.188863 & 0.597800 & 0.000000 \\
\hline 10 & 7 & 0 & 0.000000 & 1.253911 & 0.000000 \\
\hline 11 & 7 & 0 & -1.222876 & 0.703695 & 0.000000 \\
\hline
\end{tabular}


6/10- $\mathrm{H}^{+}, \mathrm{E}=-252.1111958$ a.u.

\begin{tabular}{|c|c|c|c|c|c|}
\hline \multirow{2}{*}{$\begin{array}{l}\text { Center } \\
\text { Number }\end{array}$} & \multirow{2}{*}{$\begin{array}{l}\text { Atomic } \\
\text { Number }\end{array}$} & \multirow{2}{*}{$\begin{array}{c}\text { Atomic } \\
\text { Type }\end{array}$} & \multicolumn{3}{|c|}{ Coordinates (Angstroms) } \\
\hline & & & $\mathrm{X}$ & $\mathrm{Y}$ & Z \\
\hline 1 & 6 & 0 & -1.223466 & -0.751263 & 0.000000 \\
\hline 2 & 6 & 0 & -1.155373 & 0.663813 & 0.000000 \\
\hline 3 & 7 & 0 & 1.187102 & 0.641789 & 0.000000 \\
\hline 4 & 1 & 0 & -2.211432 & -1.197150 & 0.000000 \\
\hline 5 & 1 & 0 & -2.032653 & 1.298738 & 0.000000 \\
\hline 6 & 5 & 0 & 1.284779 & -0.781199 & 0.000000 \\
\hline 7 & 1 & 0 & 2.364289 & -1.252631 & 0.000000 \\
\hline 8 & 1 & 0 & 1.983831 & 1.268811 & 0.000000 \\
\hline 9 & 6 & 0 & -0.073525 & -1.492512 & 0.000000 \\
\hline 10 & 1 & 0 & -0.158817 & -2.574135 & 0.000000 \\
\hline 11 & 7 & 0 & 0.000000 & 1.291705 & 0.000000 \\
\hline 12 & 1 & 0 & 0.035356 & 2.307680 & 0.000000 \\
\hline
\end{tabular}

7/14- $\mathrm{H}^{+}, \mathrm{E}=-252.1589068$ a.u.

\begin{tabular}{|c|c|c|c|c|c|}
\hline \multirow{2}{*}{$\begin{array}{l}\text { Center } \\
\text { Number }\end{array}$} & \multirow{2}{*}{$\begin{array}{l}\text { Atomic } \\
\text { Number }\end{array}$} & \multirow{2}{*}{$\begin{array}{l}\text { Atomic } \\
\text { Type }\end{array}$} & \multicolumn{3}{|c|}{ Coordinates (Angstroms) } \\
\hline & & & $\mathrm{X}$ & Y & Z \\
\hline 1 & 6 & 0 & 1.235165 & 0.639020 & 0.000000 \\
\hline 2 & 7 & 0 & -1.191976 & -0.678598 & 0.000000 \\
\hline 3 & 1 & 0 & 2.084707 & 1.310841 & 0.000000 \\
\hline 4 & 1 & 0 & -0.018828 & 2.299721 & 0.000000 \\
\hline 5 & 5 & 0 & 0.010297 & -1.514465 & 0.000000 \\
\hline 6 & 1 & 0 & -0.127250 & -2.684170 & 0.000000 \\
\hline 7 & 1 & 0 & -2.117303 & -1.096990 & 0.000000 \\
\hline 8 & 6 & 0 & 1.301558 & -0.712873 & 0.000000 \\
\hline 9 & 1 & 0 & 2.285165 & -1.166366 & 0.000000 \\
\hline 10 & 1 & 0 & -2.073533 & 1.228567 & 0.000000 \\
\hline 11 & 6 & 0 & -1.160159 & 0.645817 & 0.000000 \\
\hline 12 & 7 & 0 & 0.000000 & 1.285589 & 0.000000 \\
\hline
\end{tabular}


8/13- $\mathrm{H}^{+}, \mathrm{E}=-252.1489026$ a.u.

\begin{tabular}{|c|c|c|c|c|c|}
\hline \multirow{2}{*}{$\begin{array}{l}\text { Center } \\
\text { Number }\end{array}$} & \multirow{2}{*}{$\begin{array}{l}\text { Atomic } \\
\text { Number }\end{array}$} & \multirow{2}{*}{$\begin{array}{c}\text { Atomic } \\
\text { Type }\end{array}$} & \multicolumn{3}{|c|}{ Coordinates (Angstroms) } \\
\hline & & & $\mathrm{X}$ & $\mathrm{Y}$ & Z \\
\hline 1 & 7 & 0 & 0.028993 & -1.387521 & 0.000000 \\
\hline 2 & 1 & 0 & -0.070678 & 2.349704 & 0.000000 \\
\hline 3 & 1 & 0 & -2.097896 & 1.215559 & 0.000000 \\
\hline 4 & 5 & 0 & 1.308330 & -0.750279 & 0.000000 \\
\hline 5 & 1 & 0 & 2.306670 & -1.372977 & 0.000000 \\
\hline 6 & 1 & 0 & -0.033481 & -2.401529 & 0.000000 \\
\hline 7 & 6 & 0 & 1.203569 & 0.777899 & 0.000000 \\
\hline 8 & 1 & 0 & 2.034720 & 1.473382 & 0.000000 \\
\hline 9 & 1 & 0 & -2.072343 & -1.297779 & 0.000000 \\
\hline 10 & 6 & 0 & -1.154370 & -0.724662 & 0.000000 \\
\hline 11 & 6 & 0 & -1.184465 & 0.641468 & 0.000000 \\
\hline 12 & 7 & 0 & 0.000000 & 1.332778 & 0.000000 \\
\hline
\end{tabular}

9- $\mathrm{H}^{+}, \mathrm{E}=-252.1964365$ a.u.

\begin{tabular}{|c|c|c|c|c|c|}
\hline \multirow{2}{*}{$\begin{array}{l}\text { Center } \\
\text { Number }\end{array}$} & \multirow{2}{*}{$\begin{array}{l}\text { Atomic } \\
\text { Number }\end{array}$} & \multirow{2}{*}{$\begin{array}{c}\text { Atomic } \\
\text { Type }\end{array}$} & \multicolumn{3}{|c|}{ Coordinates (Angstroms) } \\
\hline & & & $\mathrm{X}$ & $Y$ & Z \\
\hline 1 & 6 & 0 & 0.000000 & 1.194622 & 0.682741 \\
\hline 2 & 6 & 0 & 0.000000 & 0.000000 & 1.386815 \\
\hline 3 & 6 & 0 & 0.000000 & -1.194622 & 0.682741 \\
\hline 4 & 7 & 0 & 0.000000 & -1.201732 & -0.661069 \\
\hline 5 & 1 & 0 & 0.000000 & 2.151706 & 1.192330 \\
\hline 6 & 1 & 0 & 0.000000 & 0.000000 & 2.466967 \\
\hline 7 & 1 & 0 & 0.000000 & -2.151706 & 1.192330 \\
\hline 8 & 5 & 0 & 0.000000 & 0.000000 & -1.450609 \\
\hline 9 & 1 & 0 & 0.000000 & 0.000000 & -2.625488 \\
\hline 10 & 7 & 0 & 0.000000 & 1.201732 & -0.661069 \\
\hline 11 & 1 & 0 & 0.000000 & 2.108974 & -1.115954 \\
\hline 12 & 1 & 0 & 0.000000 & -2.108974 & -1.115954 \\
\hline
\end{tabular}


11/15- $\mathrm{H}^{+}, \mathrm{E}=-252.0702445$ a.u.

\begin{tabular}{|c|c|c|c|c|c|}
\hline \multirow{2}{*}{$\begin{array}{l}\text { Center } \\
\text { Number }\end{array}$} & \multirow{2}{*}{$\begin{array}{l}\text { Atomic } \\
\text { Number }\end{array}$} & \multirow{2}{*}{$\begin{array}{c}\text { Atomic } \\
\text { Type }\end{array}$} & \multicolumn{3}{|c|}{ Coordinates (Angstroms) } \\
\hline & & & $\mathrm{X}$ & $\mathrm{Y}$ & Z \\
\hline 1 & 1 & 0 & -1.989906 & 1.174040 & 0.000000 \\
\hline 2 & 1 & 0 & -0.079821 & 2.277990 & 0.000000 \\
\hline 3 & 5 & 0 & 0.064134 & -1.575350 & 0.000000 \\
\hline 4 & 1 & 0 & 0.013586 & -2.753484 & 0.000000 \\
\hline 5 & 1 & 0 & 2.318952 & -1.127716 & 0.000000 \\
\hline 6 & 6 & 0 & -1.227806 & -0.728106 & 0.000000 \\
\hline 7 & 1 & 0 & -2.248806 & -1.096774 & 0.000000 \\
\hline 8 & 1 & 0 & 2.041141 & 1.328306 & 0.000000 \\
\hline 9 & 6 & 0 & 1.311161 & -0.728387 & 0.000000 \\
\hline 10 & 6 & 0 & 1.204322 & 0.638473 & 0.000000 \\
\hline 11 & 7 & 0 & 0.000000 & 1.268061 & 0.000000 \\
\hline 12 & 7 & 0 & -1.157411 & 0.586582 & 0.000000 \\
\hline
\end{tabular}

12- $\mathrm{H}^{+}, \mathrm{E}=-252.1023553$ a.u.

\begin{tabular}{|c|c|c|c|c|c|}
\hline \multirow{2}{*}{$\begin{array}{l}\text { Center } \\
\text { Number }\end{array}$} & \multirow{2}{*}{$\begin{array}{l}\text { Atomic } \\
\text { Number }\end{array}$} & \multirow{2}{*}{$\begin{array}{c}\text { Atomic } \\
\text { Type }\end{array}$} & \multicolumn{3}{|c|}{ Coordinates (Angstroms) } \\
\hline & & & $\mathrm{X}$ & $\mathrm{Y}$ & Z \\
\hline 1 & 1 & 0 & 0.000000 & 1.999894 & 1.187702 \\
\hline 2 & 1 & 0 & 0.000000 & 0.000000 & 2.389571 \\
\hline 3 & 5 & 0 & 0.000000 & 0.000000 & -1.567433 \\
\hline 4 & 1 & 0 & 0.000000 & 0.000000 & -2.746012 \\
\hline 5 & 1 & 0 & 0.000000 & -2.274192 & -1.077481 \\
\hline 6 & 6 & 0 & 0.000000 & 1.249388 & -0.723644 \\
\hline 7 & 1 & 0 & 0.000000 & 2.274192 & -1.077481 \\
\hline 8 & 1 & 0 & 0.000000 & -1.999894 & 1.187702 \\
\hline 9 & 6 & 0 & 0.000000 & -1.249388 & -0.723644 \\
\hline 10 & 6 & 0 & 0.000000 & 0.000000 & 1.310682 \\
\hline 11 & 7 & 0 & 0.000000 & -1.149427 & 0.628058 \\
\hline 12 & 7 & 0 & 0.000000 & 1.149427 & 0.628058 \\
\hline
\end{tabular}

